\title{
Diffusion Tensor Measures of the Corpus Callosum in Adolescents With Adolescent Onset Alcohol Use Disorders
}

\author{
Michael D. De Bellis, Elizabeth Van Voorhees, Stephen R. Hooper, Nicole Gibler, Lauren \\ Nelson, Steve G. Hege, Martha E. Payne, and James MacFall \\ Duke Healthy Childhood Brain Development and Developmental Traumatology Research \\ Program, Department of Psychiatry and Behavioral Sciences, Duke University Medical Center, \\ North Carolina (MDDB, EVV, SRH); Department of Psychiatry and The Clinical Center for the \\ Study of Development and Learning, University of North Carolina School of Medicine, North \\ Carolina (SRH); University of Cincinnati College of Medicine, Ohio (NG); Frank Porter Graham \\ Child Development Institute, University of North Carolina-Chapel Hill, North Carolina (LN); Rho \\ Inc., Chapel Hill, North Carolina (SGH); Department of Psychiatry and Behavioral Sciences, \\ Neuropsychiatric Imaging Research Laboratory, Duke University Medical Center, North Carolina \\ (MEP); and Department of Psychiatry, Radiology, and the Neuropsychiatric Imaging Laboratory, \\ Duke University Medical Center, North Carolina (JM).
}

\section{Abstract}

Background-In adults, myelination injury is associated with alcoholism. Maturation of the corpus callosum is prominent during adolescence. We hypothesized that subjects with adolescentonset alcohol use disorders (AUD; defined as Diagnostic and Statistical Manual of Mental Disorders-IV alcohol dependence or abuse) would have myelination mircostructural differences compared to controls.

Methods-Adolescent subjects (25 males, 7 females) with an AUD (16.9 \pm 1.2 years), who were recruited from substance abuse treatment programs and had co-morbid mental disorders, and 28 sociodemographically similar healthy controls (17 males, 11 females; $15.9 \pm 1.1$ years) underwent a 3.0 T MRI diffusion tensor imaging scan.

Results-Measures of rostral body fractional anisotropy (FA) were higher in the AUD group than in the control group. Compared to controls, mean diffusivity (MD) was lower, while FA was higher, in the AUD group in the isthmus region. Anterior corpus callosum mircostructural development differed in adolescents with AUD, as age was positively (not negatively) associated with rostrum MD and age was negatively (not positively) associated with rostrum FA. There were sex by group interactions in that control females had higher posterior midbody FA when com pared to female adolescents with AUD.

Conclusions-Lower MD and higher FA values in the AUD group suggest pre-morbid vulnerability for accelerated prefrontal and temporo-parietal myelin maturation that may enhance the risk for adolescent AUD. Significant (and opposite to developmentally expected) correlations were seen between anterior corpus callosum MD and FA measures and age in the AUD group, suggesting neurotoxic effects of alcohol on adolescent corpus callosum microstructure. As seen in adults, female adolescents with AUD may be especially vulnerable to corpus callosum mircostructural injury. Further diffusion tensor imaging studies of corpus callosum maturation in

Copyright $\odot 2008$ by the Research Society on Alcoholism.

Reprint requests: Michael D. De Bellis MD, MPH, Professor of Psychiatry and Behavioral Sciences, Director Healthy Childhood Brain Development and Developmental Traumatology Research Program, Department of Psychiatry and Behavioral Sciences, Duke University Medical Center, Box 3613, Durham NC, 27710; Fax: 919-419-0165; debel002@ mc.duke.edu. 
children at familial risk for alcoholism, and in those with AUD, need to be done to elucidate these mechanisms.

\title{
Keywords
}

Alcohol Use Disorders; Alcohol Abuse or Dependence; Diffusion Tensor Imaging; Adolescence; Corpus Callosum

\begin{abstract}
Adolescent onset alcohol use disorders (AUD), defined as DSM-IV alcohol dependence or abuse, are prevalent and serious disorders that usually continue into adulthood (Clark, 2004). In preclinical studies, excessive alcohol consumption during adolescence affects specific brain areas (frontal lobes, hippocampus, amygdala, and cerebellum) (White and Swartzwelder, 2006) and thus may adversely impact human brain maturation. In adults, alcoholism is associated with white matter volume loss (Chanraud et al., 2007) and demyelination (Lewohl et al., 2000), particularly in the corpus callosum (CC), the largest white matter structure in the cerebrum (Harper, 1998).
\end{abstract}

Diffusion tensor imaging (DTI), a non-invasive neuroimaging technique, quantifies in vivo information about the directionality and coherence of the brain's white matter tracts (Taylor et al., 2004). DTI measures the translational motion of water molecules driven by thermal energy in human tissues. In the absence of barriers, the movement of these molecules is random or isotropic. Water diffusivity is measured as mean diffusivity (MD), is greatest along fiber tracts, and suggests demyelination and axonal loss. Fractional anisotropy (FA), the deviation from randomness, is greatest in highly organized myelin tracts, and is thought to reflect myelin fiber integrity and coherence (Le Bihan et al., 2001).

Recent research using DTI demonstrated increased white matter diffusivity and decreased indices of FA (suggesting diminished axonal fiber integrity and coherence) in the anterior corpus callosum (i.e., genu) of adults with alcohol dependence (Pfefferbaum and Sullivan, 2005), as well as lower FA (Schulte et al., 2005)and higher mean diffusivity in the overall $\mathrm{CC}$ and its subregions (Pfefferbaum et al., 2007). These findings suggest that alcohol dependence may damage the microstructure of the CC. Furthermore, a recent review of nine reports published to date suggest focal disruption of commissural connectivity in the $\mathrm{CC}$ in adults with addictions (Arnone et al., 2006).

It is important to note that MD and FA measures reflect white matter integrity and maturation from childhood to adolescence. Age-appropriate myelination is reflected by decreases in MD and increases in FA measures (Morriss et al., 1999). These myelination changes parallel cognitive and affective growth, including gains in executive functions (attention, integration of higher levels information), learning and memory, and emotional regulation. Adolescent AUD may negatively impact the development of the CC and resultant cognitive processes. Alternatively, it is possible that pre-existing $\mathrm{CC}$ mircostructural damage in areas that reflect prefrontal cortical myelin fiber integrity (the rostrum and genu of the $\mathrm{CC}$ ) may be a risk factor for adolescent and adult AUD. In one study, adolescents with AUD and co-morbid mental disorders had significantly smaller prefrontal cortex (PFC) and PFC white matter volumes, and greater amounts of PFC CSF than controls (De Bellis et al., 2005).

To date, only one pilot investigation has been published investigating white matter integrity in adolescents with AUD. Using DTI and fMRI techniques, 8 adolescents with AUD demonstrated reduced FA in the posterior CC (splenium). Further FA was positively correlated with brain activation during a working memory task (Tapert and Schweinsburg, 2005). We are only just beginning to understand how excessive alcohol consumption affects 
adolescence brain and cognitive development. The absence of literature in this area is significant as adolescence is a period of unique developmental changes in overall cortical myelination, particularly the development of the $\mathrm{CC}$ and other prefrontal commissures (Berlicchi, 2003; Thompson et al., 2003).

In our previous work, we found no differences in the gross anatomical measures of the CC in adolescents and young adults with an adolescent onset-AUD (De Bellis et al., 2000a). However, in this pilot investigation, we compared more sensitive DTI measures of the CC in adolescents with AUD to those of sociodemographically similar controls. The CC was divided into 7 regions that reflect specific brain areas (De Bellis et al., 1999) for quantitative DTI measures: Region 1 (rostrum) reflects the orbital prefrontal and inferior premotor areas; Region 2 (genu), the prefrontal areas; Region 3 (rostral body), the premotor, prefrontal, and supplementary motor areas; Region 4 (anterior midbody), the motor and association regions; Region 5 (posterior midbody), the somatesthetic, posterior parietal, and association regions; Region 6 (isthmus), the superior temporal and posterior parietal association areas; and Region 7 (splenium), the occipital and inferior temporal cortical areas (De Lacoste et al., 1985; Witelson, 1989).

It was hypothesized that adolescents with AUD would show evidence of white matter mircostructural damage in the CC (higher measures of MD and lower FA) than controls. In the real world, adolescents with AUD have confounds that need to be considered in neurobiological studies of youth addiction such as familial substance use disorder, prenatal substance exposure, high rates of early life trauma, psychopathology, and co-morbidity of other substance disorders (Clark et al., 1997a; Tapert and Schweinsburg, 2005). Planned analyses were thus undertaken to examine separately these effects as well as alcohol consumption variables in our analyses. In many studies of adolescents with AUD, problematic cannabis use is common. Since the active component of marijuana, 9tetrahydrocannabinol (THC) collects in myelin (Ashton, 2001; Iversen, 2003) and affects growth factor gene expression (Mailleux et al., 1994), a comparison of cannabis use measures on the dependent variables was planned in the AUD group. Finally, since adult females with an AUD may show disproportionately negative effects on brain structure (Harper et al., 1990), CC microstructure (Arnone et al., 2006; Pfefferbaum and Sullivan, 2002) and brain function (Hommer, 2003) from excessive drinking, a comparison of sex differences was also planned.

\section{MATERIALS AND METHODS}

\section{Subjects}

Adolescents (defined as age 12 to 19 years) with an adolescent onset AUD $(n=32)$, were recruited from community treatment centers, and control participants without a history of AUD, other substance use disorders, or current Axis I mental disorders $(n=28)$, were recruited through the community. Controls were excluded if they had a major Axis I disorder in the past 6 months prior to MRI. Five of the 28 controls had previous disorders ( $n$ $=1$ major depressive episode of one month duration, $n=1$ anxiety disorder NOS, $n=1$ disruptive disorder NOS, $n=1$ enuresis, and $n=1$ past history of adjustment disorder). Criterion for exclusion from the study included (1) significant medical or neurological illness; (2) morbid obesity or failure to thrive; (3) full scale intelligence below 80 as measured by the 2 subtest (Vocabulary, Block Design) short form of the WISC-III (Wechsler Intelligence Scale for Children -III) or WAIS-III (Wechsler Adult Intelligence Scale -III) as appropriate; (4) pregnancy; (5) birth weight $<5$ lbs or severe birth complications; and (6) insufficient English skills for consenting to the protocol. This protocol was approved by Duke University Medical Center Institutional Review Board. Subjects and their legal guardian gave written informed assent/consent. Subjects, aged 18 
years or older, gave written informed consent. Subjects received monetary compensation for participation.

Groups were similar on height, weight, socioeconomic status (SES) (most fell within the upper middle socioeconomic strata), handedness, head circumference, race, sex, birth weight, and prenatal alcohol exposure. The participants in the AUD group were slightly older, were more likely to have prenatal tobacco exposure, and more likely to have a parent with a history of an alcohol or substance use disorder. Of the AUD subjects with prenatal tobacco exposure, four also had prenatal cannabis exposure and one had prenatal exposure to cocaine. None of the controls had prenatal exposure to cannabis or cocaine. See Table 1.

\section{Clinical Evaluation}

Substance use disorders and other Axis I diagnoses were determined using the K-SADS Present and Lifetime Version (Kaufman et al., 1997) with parents, subjects, and archival records as sources of information. The KSADS-PL was modified to include additional relevant information: (1) life event questions, including traumatic events from the Child and Adolescent Psychiatric Assessment (CAPA) (Angold et al., 1995) were added to the existing life event interview; (2) questions were added about disorders that were not present in the KSADS-PL (e.g., selective mutism); (3) a structured scale was used to quantify individual symptom frequency, ranging from 0 (no history of the symptom) to 10 (symptom present several times a day); and (4) algorithms or scoring forms were used to determine the presence of psychiatric and substance use disorders based on DSM-IV diagnostic criteria. This modified version of the KSADS-PL is available upon request.

Interviewers were individually trained to obtain over $90 \%$ agreement with a board certified child and adolescent psychiatrist and experienced interviewer (MDDB) for substance use disorder and for the presence of lifetime major Axis I co-morbid disorders. Discrepancies were resolved by re-interviewing the adolescent or caregiver and/or reviewing archival information. If diagnostic disagreements were not resolved with this method, consensus diagnoses were reached among the clinically experienced child psychiatrist (MDDB) and child psychologist (SRH) using the Best Estimate Method (Clark, 1999; Kosten and Rounsaville, 1992), where a date of onset, defined as the time at which diagnostic criteria were first met, was determined for each disorder (Clark et al., 2001).

Substance use information was gathered by direct interviews with the adolescents. For each symptom, age of onset was estimated to the nearest month. Methods from the Lifetime History of Alcohol Use Interview (Skinner, 1982) were incorporated into the KSADS and used to collect supplemental information on alcohol and other abused substances including cannabis, nicotine, and seven other drug classes (Stimulants, Sedatives/Hypnotics/ Anxiolytics, Cocaine, Opioids [heroin, morphine, codeine], Hallucinogens, Solvents/ inhalants, and Other [e.g., nitrous oxide, ecstasy, MDA)]). Additional information collected included average quantity and frequency of use, age of onset of regular use (defined as use of at least 2 times a month for 2 months), maximum frequency and quantity of use, and age of maximum frequency and quantity of use. Quantity variables were based on 0.6-oz. (17-g) ethanol "standard drinks" (one 12-oz. [340-g] beer, one 5-oz. [142-g] glass of table wine [12\% alcohol by volume], or one $1.5 \mathrm{oz}$. [ $42.5 \mathrm{~g}$ ] of 80 -proof hard liquor). Frequency variables were calculated as the mean number of drinks per week during the 6 months prior to enrollment in treatment. Cannabis use was translated into a standard joint (Chung et al., 2004).

The AUD group consisted of 16 adolescents with lifetime alcohol dependence (13 males, 3 females; 14 of these with dependence had a prior history of alcohol abuse), and 16 adolescents with current alcohol abuse. The mean age of onset for alcohol dependence was 
$15.3 \pm 1.37$ years with a mean duration of $0.80 \pm 1.24$ years. The mean age of onset for alcohol abuse was $14.7 \pm 1.34$ years with a mean duration of $1.25 \pm 0.79$ years. Thus, the combined mean age of onset for an AUD was $14.7 \pm 1.33$ years (with an age range of 12 to 17 years) and with a mean duration of $2.05 \pm 1.1$ years (with a range of 0.67 to 6.25 years). In the AUD group, regular drinking began at a mean age of $15.6 \pm 1.3$ years with a range of 12 to 17 years, and the mean number of drinks per week was $12.9 \pm 20.0$ drinks. In the AUD group, the mean number of drinks per maximum drinking episode was $13.2 \pm 8.6$, with a range of 3.3 to 41 drinks. The mean age of the maximum drinking episode was $15.6 \pm 1.3$ years, with a range of 12 to 17.6 years. The mean days since the AUD subjects last consumed alcohol was $63.7 \pm 88.2$ days. Ten of the 32 AUD subjects had a history of at least one DSM-IV alcohol withdrawal symptom.

Comorbid cannabis use disorders and nicotine dependence were common among the adolescents with AUD. Twenty-nine of the 32 AUD subjects tried cannabis, and 31 of the 32 AUD subjects tried cigarettes. Twenty-four met criteria for a cannabis use disorder (CUD): 2 met criteria for lifetime cannabis abuse, and 22 met criteria for lifetime cannabis dependence (18 of these had a prior history of cannabis abuse). The mean age of onset for cannabis dependence was $15.4 \pm 1.17$ years with a mean duration of $2.49 \pm 1.38$ years. The mean age of onset for cannabis abuse was $14.4 \pm 1.54$ years, with a mean duration of $1.0 \pm$ 0.62 years. Thus, the combined mean age of onset for a CUD was $14.5 \pm 1.53$ years (with an age range of 11 to 17 years), and with a mean duration of $2.3 \pm 1.3$ years, (with a range of 0.5 to 6.25 years). In the CUD group, the mean number of joints per maximum use episode was $22.5 \pm 26.6$ joints. The mean age of the maximum use episode was $15.6 \pm 1.3$ years, with a range of 12 to 17.6 years. Among the subset with AUD+CUD, the mean days since subjects last used cannabis was $130.3 \pm 260.7$ days. Nine of the 24 AUD with CUD subjects had a history of at least one DSM-IV cannabis withdrawal symptom.

Twelve of the 32 adolescents with AUD met criteria for nicotine dependence. The mean number of cigarettes used per day was $8.2 \pm 9.8$ cigarettes. Other substance use disorder comorbidities included: stimulant abuse $(N=3)$, stimulant dependence $(N=1)$, sedative abuse $(n=1)$, cocaine abuse $(n=3)$, cocaine dependence $(n=2)$, opioids abuse $(n=3)$, and opioids dependence $(n=1)$.

Co-morbidity is common in adolescent AUD, with most adolescents with AUD having at least one other co-morbid mental disorder (Agosti et al., 2002; Clark et al., 1997a,b; Grella et al., 2001). In our sample, AUD subjects were classified as having a mood disorder if they received a lifetime classification of major depressive disorder (MDD) $(N=23)$, dysthymia $(N=6)$, depressive disorder not otherwise specified (NOS) or adjustment disorder with depressed mood $(N=4)$. Eighty-three percent of the AUD sample had some type of mood disorder. Conduct disorder (CD) $(N=20)$, oppositional defiant disorder (ODD) $(N=24)$, and disruptive disorders NOS $(N=0)$ were combined similarly, resulting in $83 \%$ of the AUD sample having some form of disruptive behavior disorder. Finally, those with a classification of generalized anxiety disorder (GAD) $(N=7)$, separation anxiety disorder (SAD) $(N=1)$, panic disorder $(N=1)$, anxiety disorder NOS $(N=0)$, or obsessivecompulsive disorder (OCD) $(N=1)$ were assigned an anxiety disorder classification. Approximately, $30 \%$ of the AUD sample experienced some form of anxiety disorder. A comorbid Axis I index was then computed as the sum of the following: mood disorder classification, disruptive behavior disorder classification, anxiety disorder classification, posttraumatic stress disorder (PTSD) $(N=4)$, attention-deficit hyperactivity disorder (ADHD) $(N=22)$, tic disorder $(N=1)$, enuresis $(N=5)$, encopresis $(N=2)$, and adjustment disorder (AD) $(N=8)$. For the AUD group the mean of the Axis I index was 3.2, with a standard deviation of 1.1, and a range from 1 to 6 . 
Because adverse life events are common in adolescents with AUD (Clark et al., 1997a), an early trauma index (ETI) was computed as the sum of the affirmative responses given about experiencing (including witnessing or hearing about a experience of a close friend or family member) the following traumatic events: sexual abuse, auto accident, fire, disaster, neglect, other accident, violent crime, family violence, emotional abuse, physical abuse, corporal punishment, traumatic news, and family illness. The AUD group [Mean $(\mathrm{SD})=7.7(2.2)$ ] experienced significantly more traumatic events than the control group (Mean (SD) = 4.9(1.8), $t(77)=-6.1, p<0.001)$. Subjects in the AUD group were more likely to have been reported to child protective services for neglect or abuse $(N=11$, FTE $p=0.0004)$, to have witnessed family violence $(N=24$, FTE $p=0.0002)$, or to have been a victim of, or witness to, a violent crime $\left(X^{2}=15.2, p<0.0001\right)$.

\section{Magnetic Resonance Imaging}

Magnetic resonance imaging (MRI) was performed using a Siemens Trio 3.0 Tesla MRI system (Trio, Siemens Medical Systems) running version VA 24 software located at the Duke University Medical Center (DUMC) Department of Radiology. Diffusion weighted images (DWI) were acquired using a single-shot echo-planar imaging (EPI) pulse sequence. Imaging parameters were $\mathrm{TE}=90 \mathrm{mseconds}, \mathrm{TR}=160$ mseconds (for 45 slices $\mathrm{TR}=7200$ mseconds), Bandwidth $1346 \mathrm{~Hz} /$ pixel, acquisition matrix $=128 \times 64$, FOV $=220 \mathrm{~mm}$, 3$\mathrm{mm}$ slice thickness. Images were acquired with diffusion weighting in each of 6 different directions, all with b-values (diffusion weighting factor) of 0 , 1000. In addition, an image with no diffusion weighting (b-value of 0 ) was acquired as reference. The set of seven DWI were acquired a total of 4 times for retrospective averaging to improve image quality. The four sets of DWI were averaged together after the magnitude-image reconstruction, and the diffusion tensor eigenvalues were calculated in each voxel allowing the calculation of the MD and the FA values in each voxel using established methods (Taylor et al., 2004). Briefly, the diffusivity in a voxel is modeled as a tensor. The seven DTI measurements of the intensity for each voxel are used to calculate the three eigenvalues (D1, D2, D3) and corresponding eigenvectors of the tensor for that voxel. The MD is then calculated as the average of the eigenvalues and the FA as FA $=$ SQRT[(3/2) $\left((\mathrm{D} 1-\mathrm{MD})^{2}+(\mathrm{D} 2-\mathrm{MD})^{2}+(\mathrm{D} 3-\right.$ $\left.\left.\mathrm{MD})^{2}\right) /\left(\mathrm{D} 1^{2}+\mathrm{D} 2^{2}+\mathrm{D} 3^{2}\right)\right]$. Scans with clinically significant abnormalities were dropped from the analyses $(n=0)$. Subjects tolerated the procedure well. No sedation was used.

Image analysts who were blind to subject group and trained by the authors (MEP, JM, MDDB) selected corpus callosum regions-of-interest (ROIs) placed on the $b=0$ image. The corresponding ROI's for the ADC and FA images were used for calculating MD and FA values. ROIs consisted of approximately $0.5-\mathrm{cm}^{3}$ regions placed in each of the 7 divisions of the corpus callosum divided as previously described (De Bellis et al., 1999). For the purposes of obtaining excellent reliability, two raters concurrently evaluated the seven regions for analyses, thus intra-rater ICC values were MD rostrum, 0.99; MD genu, 0.99; MD rostral body, 0.99; MD anterior midbody, 0.94; MD posterior midbody, $0.98 \mathrm{MD}$ isthmus, 0.84; MD splenium, 0.99; and for FA intra-Rater ICC values were rostrum, 0.98; genu, 0.97; rostral body, 0.97; anterior midbody, 0.99; posterior midbody, 0.99 isthmus, 0.84; splenium, 0.99 .

\section{Statistical Methods}

Distributions of the DTI and clinical measures were examined for normality using Shapiro and Wilk's W-statistic. Residuals were inspected in cases where the dependent data distributions were not normal. For these measures typical parametric procedures were determined to be adequately robust (Kleinbaum et al., 1988). Background characteristics on the AUD and control groups were compared using $t$-tests, FET, or $\chi^{2}$ for categorical measures. Significantly different background characteristics (i.e., age) were used as 
covariates to statistically balance the two groups and improve efficiency of the estimates. Covariates for all analyses involving the DTI measures initially included for age, IQ and then age and sex. Separate regression analyses using general linear models were conducted to examine group differences between the AUD and control group in the DTI adjusting for covariates, and to estimate associations of predictors of interest. Preliminary analyses were performed to determine whether there were significant group interactions with covariates and predictors of interest (i.e., group by age, group by IQ, group by sex, and group by predictor (e.g., ETI, co-morbid disorders). When significant, these interactions were retained in subsequent analyses. A follow-up analysis was conducted to determine if there were differences in adolescents having both AUD and CUD (cannabis abuse or dependence) verses AUD only adolescents on the DTI measures.

Effect size estimates of the differences between the AUD and control groups were computed as the adjusted mean for AUD minus the adjusted mean in the control group divided by the standard deviation for the DTI measure in the control group. Partial correlations (i.e., $r_{\mathrm{p}}$, correlations adjusted for covariates) are reported as unadjusted and adjusted effect size estimates of the associations between a predictor of interest (i.e., ETI, age of onset, duration of AUD, consumption) and the DTI measures.

\section{RESULTS \\ DTI Measures}

As can be seen in Table 2, we did not find the hypothesized increase in MD or decrease in FA in CC regions in the comparison between the adolescent AUD and control groups.

\section{Main Effects of Group}

Contrary to our hypotheses, FA values in the CC rostral body were higher in the AUD group than in the control group, and the difference remained significant after adjusting for age $(F(1,55)=4.2, p<0.05)$, but not after adjusting for age and $\operatorname{sex}(F(1,55)=2.9, p=0.09)$. In both adjusted analyses, effect sizes were within the moderate range ( $d=0.4$ to 0.52 ). MD in the $\mathrm{CC}$ isthmus was lower in the AUD group than in the control group after adjusting for age, for age and sex, and for IQ $(F=7.7, p<0.008)$. Effect sizes were large for these analyses $(d=0.8)$. FA values in the CC isthmus were higher in the AUD group (Mean $=$ 573.6) than in the controls (Mean $=529.8)$ after adjusting for age $(d=0.7)$, age and sex $(d=$ $0.9)$, and IQ $(F=12.6, p=0.0008)$. Further, FA values in the rostral body were highest in the AUD subjects with cannabis dependence $(F=3.658, p=0.03)$ compared to controls (contrasts $p$-value $=0.01$ ); and the MD in the isthmus region was lowest for the AUD subjects with cannabis dependence $(F=3.02, p=0.06)$ compared to controls (contrasts $p$ value $=0.02$ ). These findings may reflect accelerated CC rostral body (reflecting the PFC) and $\mathrm{CC}$ isthmus (reflecting the superior temporal and posterior parietal association areas) mircostructural maturation, which may, in turn, be a neurobiological marker of enhanced risk for adolescent substance use disorders.

\section{The Main Effects of Clinical Factors Within the AUD Group}

AUD Age of Onset-Age of AUD onset was positively associated with MD in the isthmus $\left(r_{\mathrm{p}}=0.52, F(1,28)=5.8, p<0.05\right)$ and negatively associated with FA in the anterior midbody region $\left(r_{\mathrm{p}}=-0.57, F(1,28)=7.0, p<0.05\right)$. Thus, the lower the MD and the higher the FA in the CC isthmus, the younger the age of onset of an AUD indicating that lower CC isthmus MD may be a risk factor for AUD in youth. Also, the older the adolescents met criteria for an AUD, the higher the MD value, suggesting alcohol-related mircostructural abnormalities in adolescents with later-onset drinking problems. 
Duration of AUD and AUD Clinical Measures-Duration of alcohol use was negatively associated with MD in the isthmus $\left(r_{\mathrm{p}}=-0.42 ; F(1,28)=5.0, p<0.05\right)$, and positively associated with FA in the CC anterior midbody $\left(r_{\mathrm{p}}=0.43 ; F(1,28)=4.9, p<\right.$ $0.05)$. Contrary to hypotheses, these findings suggest that the longer the use of alcohol, the lower the MD value in the isthmus and the higher the FA value in the CC anterior midbody, an area that reflects motor and association regions of the cerebrum.

Mean days since last consumed alcohol was negatively associated with MD in the CC Rostral Body $\left.\left(r_{\mathrm{p}}=\right) 0.33, F(1,28)=4.3, p<0.05\right)$ and CC anterior midbody $\left(r_{\mathrm{p}}=0.41\right.$, $F(1,28)=5.9, p<0.05)$ regions. Number of drinks reported was negatively associated with $\mathrm{MD}$ in the Rostral Body region $\left(r_{\mathrm{p}}=-0.37, F(1,28)=4.6, p<0.05\right)$.

There was no evidence for associations among ethanol withdrawal symptoms and the DTI measures. Overall, these findings may indicate that lower MD in specific CC subregions is a risk factor for AUD in youth.

\section{Group by Age Interactions}

Since the developmental literature suggests that in the CC MD should decrease and CC FA should increase with age, the associations between the DTI measures and age were also of interest. When examining all subjects, the predicted significant group by age interactions were found in the rostrum [for MD, $F(1,55)=5.98, p=0.02$; and for FA, $F(1,55)=4.94, p<$ $0.03]$, and splenium [MD, $F(1,58)=8.4 p<0.05]$ regions.

However, in this cross-sectional data, group by age interactions found in the control group were as expected, with MD values decreasing and FA values increasing with age. In the rostrum, age was negatively associated with $\mathrm{MD}\left(r_{\mathrm{p}}=-0.28, p<0.05\right)$ and positively associated with FA in the control group $\left(r_{\mathrm{p}}=0.28, p<0.05\right)$. However, group by age interactions in the AUD group were opposite of what would have been predicted based on the developmental literature. In the AUD group age was positively associated with $\mathrm{MD}\left(r_{\mathrm{p}}=\right.$ $0.40, p<0.05)$ and negatively associated with FA in the rostrum $\left.\left(r_{\mathrm{p}}=\right) 0.34, p<0.05\right)$. See Figs 1 and 2.

In the splenium region, age was not significantly correlated with MD in the AUD group, but was negatively associated in the control group $\left(r_{\mathrm{p}}=-0.52, p<0.05\right)$. See Fig. 3 . These findings suggest developmental differences between the AUD and control groups in rostrum and CC splenium mircostructural maturation in subregions of the CC that reflect orbital prefrontal and inferior premotor (rostrum) and the occipital and inferior temporal cortical areas (splenium). These results may be related to direct neurotoxic effects of alcohol on developing adolescent CC macrostructure.

AUD and Co-morbid Cannabis-Use disorders. In the subgroup of adolescents with AUD and co-morbid CUD, co-morbidity was negatively associated with MD in the CC anterior midbody $\left(r_{\mathrm{p}}=-0.54, F(1,28)=9.4, p<0.05\right)$ and the CC posterior midbody $\left(r_{\mathrm{p}}=\right.$ $-0.47, F(1,28)=7.1, p<0.05)$. These findings further extend the above findings and may indicate that lower MD in specific CC subregions is a risk factor for substance use disorder in youth.

In the subgroup of adolescents with AUD and co-morbid cannabis dependence $(n=22)$, positive spearman correlations between CC MD and age were found in the rostral body ( $r=$ $0.58, p=0.004)$, anterior midbody $(r=0.59, p=0.004)$, and splenium $(r=0.576, p=0.005)$. These associations persisted when adjusting for multiple analyses. These findings further extend the findings above, and may be related to direct neurotoxic effects of alcohol and cannabis on the normal age-related development of adolescent CC macrostructure. 
Cannabis abuse age of onset, cannabis abuse or dependence duration, number of cannabis dependence withdrawal symptoms, and days since last use of cannabis were not significantly associated with any DTI measures.

\section{The Effects of Comorbid Mental Health Disorders, Familial History, Gender, and Early Trauma}

Separate regression analyses were used to assess the impact of the more frequently occurring Axis I disorders, the composite variables (mood disorder classification, disruptive behavior disorders, anxiety disorder classification), PTSD, ADHD, and Adjustment Disorder (AD), the Axis I summary index, and ETI on the DTI measures.

Having a mood disorder classification was positively correlated with $\mathrm{MD}\left[r_{\mathrm{p}}=0.45, F(1,28)\right.$ $=6.8, p<0.05]$ and negatively correlated with FA $\left[r_{\mathrm{p}}=-0.40, F(1,28)=5.0, p<0.06\right]$ in the $\mathrm{CC}$ splenium region. ADHD was negatively correlated with MD in the rostrum $\left[r_{\mathrm{p}}=\right.$ $-0.37, F(1,28)=4.1, p<0.05]$.

Across the AUD and control groups, we found positive associations between ETI and MD in the genu region $\left[r_{\mathrm{p}}=0.41, F(1,55)=6.8, p<0.05\right]$, thus the more traumatic events an adolescent experienced the more disorganized the microstructure of the CC genu, an area that ultimately connects to, and likely reflects PFC.

Compared to females, the males had higher FA in the rostral body $\left(r_{\mathrm{p}}=0.63\right)$ but lower FA in the isthmus $\left(r_{\mathrm{p}}=-0.81\right)$ and the splenium regions $\left(r_{\mathrm{p}}=-0.67\right)$. There were sex by group interactions in that control females had higher FA values in the posterior midbody, $[F(1,59)$ $=6.8, p<0.01]$, when compared to female adolescents with AUD.

There were no significant associations of DTI values with parental history of a substance use disorder or prenatal substance exposure.

\section{DISCUSSION}

In this pilot study we did not find the hypothesized increase in MD or decrease in FA values in the CC of adolescents with an adolescent-onset AUD. In fact, our findings were counter to our hypothesis in several important areas. In the rostral body, an anterior CC region facilitating information transfer between the prefrontal cortices and, thus involved in executive functioning processes, we found significantly increased FA and significantly decreased MD (though these effects were no longer significant when controlling for age and sex). The isthmus is a posterior CC region, and is likely involved in the transfer of information between the hemispheres of the temporo-parieto-occipital junction (an area of the association cortex which contains the angular gyrus). In this area, we found lower MD and higher FA in the AUD group, and these correlations remained when controlling for age, sex, and IQ.

Taken together, these findings suggest that the $\mathrm{CC}$ microstructure of the rostral body and the isthmus differs from controls and may be reflecting early mircostructural $\mathrm{CC}$ myelination patterns in the AUD group relative to controls. This effect may be even more pronounced in adolescents with AUD and co-morbid CUD. These results differ from DTI findings in adults with alcohol dependence, where MD has been found to be increased and FA decreased in the CC, reflecting CC mircostructural damage (Pfefferbaum et al., 2007). Our results differ from another pilot study of young adults where no differences were found between cannabis users and controls in CC FA (Gruber and Yurgelun-Todd, 2005). This unexpected directionality of the findings are especially striking because growth of the isthmus is 
prominent during childhood and adolescence (Rajapakse et al., 1996), at least theoretically making it more vulnerable to alcohol (and cannabis) related toxicity.

An explanation for these discrepant findings between adults with AUD and adolescents with AUD is that a pre-morbid vulnerability for accelerated PFC and temporo-parietal maturation enhances the risk for adolescent substance use disorders. Subjects with an adolescent onset AUD may have an inherent myelination pattern defect in prefrontal and association cortex, which could be an early risk factor for teenage addiction. In other words, it is possible that accelerated myelination of these regions may be less than optimal and may represent a risk factor for an adolescent onset substance use disorder.

This factor as well as direct neurotoxic effects of alcohol (and co-morbid cannabis) consumption on CC macrostructure may explain these complex group by age CC DTI findings. In support of this interpretation, our cross-sectional data suggest that the development of the CC microstructure in adolescents with adolescent onset-AUD differs from controls.

The rostrum is associated with connectivity in the prefrontal cortical regions, and other studies have shown decreased prefrontal white matter in adolescents and young adults with an AUD (De Bellis et al., 2005). In our AUD sample, age was positively (not negatively) associated with MD values in the rostrum region and age was negatively (not positively) associated with FA in the rostrum region. In the splenium region, a region where growth is most prominent from childhood and adolescence (Rajapakse et al., 1996), age was not significantly correlated with MD in the AUD group as would have been expected. One possible explanation for these findings is alcohol and/or cannabis related toxicity.

The splenium region is the last region to completely myelinate, with complete maturation occurring in young adulthood (Giedd et al., 1999). The direct neurotoxic effects of alcohol may be interfering with splenium maturation. Also consistent with this interpretation, CUD co-morbidity was negatively associated with $\mathrm{MD}$ in the anterior midbody region. Cannabis collects in myelin (Ashton, 2001; Iversen, 2003) and has been found to affect growth factor gene expression in the adult cortex (Mailleux et al., 1994). In our sample, we found that those subjects with AUD and co-morbid cannabis dependence did not show the normal age related decrease in MD: the correlations between MD and age were positive (not negative) in the rostral body, anterior midbody, and splenium. These results may reflect an overall negative developmental effect on the CC microstructure due to co-morbid cannabis related toxicity.

It may be that early onset AUD is associated with premature myelination, a risk factor for early addiction, and that our findings are the result of this process and of the direct neurotoxic effects of cannabis and alcohol on CC macrostructure. Our data further support this possibility in that the duration of alcohol use was negatively associated with MD in the isthmus and positively associated with FA in the anterior mid-body region. Our findings that higher MD values were associated with an older onset of AUD, older onset of regular drinking, and older age of maximum drinking episode suggest alcohol related mircostructural damage may be more likely in older adolescent drinkers. DTI studies of white matter maturation in children at familial risk for alcoholism need to be done to elucidate these processes.

The genu is a region of the $\mathrm{CC}$ that has been demonstrated to be completely myelinate early in life (Giedd et al., 1999); we found that across both the AUD and control groups, greater number of early traumatic life events was associated with higher MD values in this region in adolescents. Also in both groups, meeting criteria for a mood disorder was positively correlated with MD and negatively correlated with FA in the splenium region, a posterior 
area of the CC that completes myelination later in life. As depression is known to be a disorder of chronic or severe stress, these findings suggest that adolescents who have experienced early life trauma (regardless of substance abuse status) may demonstrate stunted or delayed CC development. Such an interpretation is consistent with the growing literature demonstrating the adverse effects of early life stress on multiple brain areas, including the prefrontal cortex (De Bellis et al., 1999, 2000b).

Further, we found ADHD to be negatively correlated with MD in the rostral region. Since anatomical studies have found decreased CC area in the rostrum region (Giedd et al., 1994), this finding may indicate accelerated myelination of the rostrum region. This accelerated process may be less than optimal for the developing brain, and subsequently may serve as a risk factor for ADHD and adolescent addictive behaviors. In addition, the contiguous relationship of the rostrum with PFC also implies the presence of deficits or dysfunction in frontal-executive abilities in these populations. Because of the substantial co-morbidity of our AUD subjects with trauma histories and mood disorders, these clinical confounds may have influenced our findings and limit the interpretation of our data.

Overall, we found sex differences in the microstructure of the CC: males had higher FA in the rostral body, but lower FA in the isthmus and the splenium regions. Other studies have found sex differences in the anatomical growth of cortical white matter, the CC (De Bellis et al., 2001), and white matter microstructure in adolescents, including higher left anterior white matter FA in males compared with females(Silveri et al., 2006). Other studies have shown decreased FA in the CC of females as compared to males (Shin et al., 2005; Westerhausen et al., 2004). However, sex differences in DTI measures of the CC were not seen in one study of adults, aged 21 to 73 years (Ota et al., 2006). In our data, sex by group interactions were found in that control females had higher FA values in the posterior midbody. This suggests that female adolescents with AUD may be especially vulnerable to the neurotoxic affects of excessive alcohol intake. These data are consistent with the adult literature where females with AUD seem to show disproportionately negative effects on brain structure (Harper et al., 1990), CC microstructure (Pfefferbaum and Sullivan, 2002), and brain function (Hommer, 2003) as compared to adult males with AUD. It should be noted that having a biological parent with a history of addiction or prenatal substance exposure did not influence our results.

The findings reported herein must be considered in light of some limitations. Our study is cross sectional and must be considered preliminary. Because little work has been done examining corpus callosum integrity in adolescents with substance use disorders, our control group was, by design, screened for the absence of psychopathology. However, in our analyses we controlled for differences in age and examined gender, the effects of higher levels of co-morbid disorders, and trauma histories in the AUD group.

In summary, our DTI findings suggest that premature myelination in the CC rostral body and isthmus reflecting PFC, superior temporal, and posterior parietal association areas may be a risk factor for early addiction. Our data also suggest that the development of the CC microstructure in adolescents with adolescent onset-AUD differs from controls. These findings may be the result of early neurobiological risk processes, co-morbidity, and/or the dynamic direct neurotoxic effects of substance use disorder on adolescent CC macrostructure. Further DTI studies of white matter maturation in children at familial risk for alcoholism, and studies involving larger samples of adolescents with AUD, are needed to elucidate these mechanisms. 


\section{Acknowledgments}

Supported in part by NIAAA grant R01 AA12479, NIDA grant RO1DA020989 and NIMH grant K24 MH071434 (PI: Dr. De Bellis).

\section{REFERENCES}

Agosti V, Nunes E, Levin F. Rates of psychiatric comorbidity among U.S. residents with lifetime cannabis dependence. Am J Drug Alcohol Abuse. 2002; 28:643-652. [PubMed: 12492261]

Angold A, Prendergast M, Cox A, Harrington R, Simonoff E, Rutter M. The Child and Adolescent Psychiatric Assessment (CAPA). Psychol Med. 1995; 25:739-753. [PubMed: 7480451]

Arnone D, Abou-Saleh MT, Barrick TR. Diffusion tensor imaging of the corpus callosum in addiction. Neuropsychobiology. 2006; 54:107-113. [PubMed: 17108711]

Ashton CH. Pharmacology and effects of cannabis: a brief review. Br J Psychiatry. 2001; 178:101106. [PubMed: 11157422]

Berlicchi, B. The midline fusion hypothesis is all right but cannot explain all callosal functions. In: Zaidel, E.; Iacoboni, M., editors. The Parallel Brain: The Cognitive Neuroscience of the Corpus Callosum. The MIT Press; London England: 2003. p. 154-156.

Chanraud S, Martelli C, Delain F, Kostogianni N, Douaud G, Aubin HJ, Reynaud M, Martinot JL. Brain morphometry and cognitive performance in detoxified alcohol-dependents with preserved psychosocial functioning. Neuropsychopharmacology. 2007; 32:429-438. [PubMed: 17047671]

Chung T, Martin CS, Winters KC, Cornelius JR, Langenbucher JW. Limitations in the Assessment of DSM-IV Cannabis Tolerance as an Indicator of Dependence in Adolescents. Exp Clin Psychopharmacol. 2004; 12:136-146. [PubMed: 15122958]

Clark, DB. Psychiatric Assessment. In: Ott, PJ.; Tarter, RE.; Ammerman, RT., editors. Sourcebook on Substance Abuse: Etiology, Epidemiology, Assessment, and Treatment. Allyn \& Bacon; Needham Heights, Massachusetts: 1999. p. 197-211.

Clark DB. The natural history of adolescent alcohol use disorders. Addiction. 2004; 99:5-22. [PubMed: 15488102]

Clark DB, Lesnick L, Hegedus A. Trauma and other stressors in adolescent alcohol dependence and abuse. J Am Acad Child Adolesc Psychiatry. 1997a; 36:1744-1751. [PubMed: 9401336]

Clark DB, Pollock N, Bukstein OG, Mezzich AC, Bromberger JT, Donovan JE. Gender and comorbid psychopathology in adolescents with alcohol dependence. J Am Acad Child Adolesc Psychiatry. 1997b; 36:1195-1203. [PubMed: 9291720]

Clark DB, Pollock NK, Mezzich A, Cornelius J, Martin C. Diachronic assessment and the emergence of substance use disorders. J Child Adolescent Substance Abuse. 2001; 10:13-22.

De Bellis MD, Clark DB, Beers SR, Soloff P, Boring AM, Hall J, Kersh A, Keshavan MS. Hippocampal Volume in Adolescent Onset Alcohol Use Disorders. Am J Psychiatry. 2000a; 157:737-744. [PubMed: 10784466]

De Bellis MD, Keshavan MS, Beers SR, Hall J, Frustaci K, Masalehdan A, Noll J, Boring AM. Sex differences in brain maturation during childhood and adolescence. Cereb Cortex. 2001; 11:552557. [PubMed: 11375916]

De Bellis MD, Keshavan M, Clark DB, Casey BJ, Giedd J, Boring AM, Frustaci K, Ryan ND. A.E. Bennett Research Award. Developmental Traumatology, Part II: Brain Development. Biol Psychiatry. 1999; 45:1271-1284. [PubMed: 10349033]

De Bellis MD, Keshavan MS, Spencer S, Hall J. N-acetylaspartate concentration in the anterior cingulate in maltreated children and adolescents with PTSD. Am J Psychiatry. 2000b; 157:11751177. [PubMed: 10873933]

De Bellis MD, Narasimhan A, Thatcher DL, Keshavan MS, Soloff P, Clark DB. Prefrontal Cortex, Thalamus and Cerebellar Volumes in Adolescents and Young Adults with Adolescent Onset Alcohol Use Disorders and Co-Morbid Mental Disorders. Alcohol: Clin Exp Res. 2005; 29:15901600. [PubMed: 16205359]

De Lacoste MC, Kirkpatrick JB, Ross ED. Topography of the human corpus callosum. J Neuropathol Exp Neurol. 1985; 44000. 
Giedd JN, Blumenthal J, Jeffries NO, Rajapakse JC, Vaituzis AC, Liu H, Tobin M, Nelson J, Castellanos X. Development of the human corpus callosum during childhood and adolescence: a longitudinal MRI study. Prog NeuroPsychopharmacol Biol Psychiatry. 1999; 23:571-588. [PubMed: 10390717]

Giedd JN, Castellanos FX, Casey BJ, Kozuch BA, King AC, Hamburger SD, Rapoport JL. Quantitative morphology of the corpus callosum in attention deficit hyperactivity disorder. Am J Psychiatry. 1994; 151:665-669. [PubMed: 8166306]

Grella CE, Hser YI, Joshi V, Rounds-Bryant J. Drug treatment outcomes for adolescents with comorbid mental and substance use disorders. J Nerv Ment Dis. 2001; 189:384-392. [PubMed: 11434639]

Gruber SA, Yurgelun-Todd DA. Neuroimaging of marijuana smokers during inhibitory processing: a pilot investigation. Cognitive Brain Res. 2005; 23:107-118.

Harper CG. The neuropathology of alcohol-specific brain damage, or does alcohol damage the brain? J Neuropathol Exp Neurol. 1998; 57:101-110. [PubMed: 9600202]

Harper CG, Smith NA, Kril JJ. The effects of alcohol on the female brain: a neuropathological study. Alcohol: Clin Exp Res. 1990; 25:445-448.

Hommer DW. Male and female sensitivity to alcohol-induced brain damage. Alcohol Res \& Health: J Natl Inst Alcohol Abuse \& Alcohol. 2003; 27:181-185.

Iversen L. Cannabis and the brain. Brain. 2003; 126:1252-1270. [PubMed: 12764049]

Kaufman J, Birmaher B, Brent D, Rao U, Flynn C, Moreci P, Williamson D, Ryan N. Schedule for affective Disorders and Schizophrenia for school-age children-present and lifetime version (KSADS-PL): Initial reliability and validity data. J Am Acad Child Adolesc Psychiatry. 1997; 36:980-988. [PubMed: 9204677]

Kleinbaum, DG.; Kupper, L.; Muller, KE. Applied Regression Analysis and Other Multivariable Methods. 2nd edition. PWS-Kent Publishing Company; Boston MA: 1988.

Kosten TA, Rounsaville BJ. Sensitivity of psychiatric diagnosis based on the best estimate procedure. Am J Psychiatry. 1992; 149:1225-1227. [PubMed: 1503136]

Le Bihan D, Mangin J-F, Poupon C, Clark CA, Pappata S, Molko N, Chabriat H. Diffusion tension imaging: concepts and applications. J Magn Reson Imaging. 2001; 13:534-546. [PubMed: 11276097]

Lewohl J, Wang L, Miles M, Zhang L, Dodd P, Harris R. Gene expression in human alcoholism: microarray analysis of frontal cortex. Alcohol Clinical and Experimental Research. 2000; 24:1873-1882.

Mailleux P, Preud'homme X, Albala N, Vanderwinden JM, Vanderhaeghen JJ. delta-9Tetrahydrocannabinol regulates gene expression of the growth factor pleiotrophin in the forebrain. Neurosci Lett. 1994; 175:25-27. [PubMed: 7970205]

Morriss MC, Zimmerman RA, Bilaniuk LT, Hunter JV, Haselgrove JC. Changes in brain water diffusion during childhood. Neuroradiology. 1999; 41:929-934. [PubMed: 10639670]

Ota M, Obata T, Akine Y, Ito H, Ikehira H, Asada T, Suhara T. Age-related degeneration of corpus callosum measured with diffusion tensor imaging. Neuroimage. 2006; 31:1445-1452. [PubMed: 16563802]

Pfefferbaum A, Rosenbloom MJ, Adalsteinsson E, Sullivan EV. Diffusion tensor imaging with quantitative fibre tracking in HIV infection and alcoholism comorbidity: synergistic white matter damage. Brain. 2007; 130:48-64. [PubMed: 16959813]

Pfefferbaum A, Sullivan EV. Microstructural but not macrostructural disruption of white matter in women with chronic alcoholism. Neuroimage. 2002; 15:708-718. [PubMed: 11848714]

Pfefferbaum A, Sullivan EV. Disruption of Brain White Matter Microstructure by Excessive Intracellular and Extracellular Fluid in Alcoholism: Evidence from Diffusion Tensor Imaging. Neuropsychopharmacology. 2005; 30:423-432. [PubMed: 15562292]

Rajapakse JC, Giedd JN, Rumsey JM, Vaituzis AC, Hamburger SD, Rapoport JL. Regional MRI measurements of the corpus callosum: a methodological and developmental study. Brain Dev. 1996; 18:379-88. [PubMed: 8891233] 
Schulte T, Sullivan EV, Muller-Oehring EM, Adalsteinsson E, Pfefferbaum A. Corpus callosal microstructural integrity influences interhemispheric processing: A diffusion tensor imaging study. Cereb Cortex. 2005; 15:1384-1392. [PubMed: 15635059]

Shin YW, Kim DJ, Ha TH, Park HJ, Moon WJ, Chung EC, Lee JM, Kim IY, Kim SI, Kwon JS. Sex differences in the human corpus callosum: diffusion tensor imaging study. Neuroreport. 2005; 16:795-798. [PubMed: 15891572]

Silveri MM, Rohan ML, Pimentel PJ, Gruber SA, Rosso IM, Yurgelun-Todd DA. Sex differences in the relationship between white matter microstructure and impulsivity in adolescents. Magn Reson Imaging. 2006; 24:833-841. [PubMed: 16916700]

Skinner, H. Development and Validation of a Lifetime Alcohol Consumption Assessment Procedure. Addiction Research Foundation; Toronto: 1982.

Tapert, SF.; Schweinsburg, AD. The human adolescent brain and alcohol use disorders. In: Galanter, M., editor. Recent Developments in Alcoholism Vol. XVII Research on Alcohol Problems in Adolescents and Young Adults. Kluwer Academic; Norwell, Massachusetts: 2005. p. 177-197.

Taylor WD, Hsu E, Krishnan RR, MacFall JR. Diffusion tensor imaging: background, potential, and utility in psychiatric research. Biol Psychiatry. 2004; 55:201-207. [PubMed: 14744459]

Thompson, PM.; Narr, KL.; Blanton, RE.; Toga, AW. Mapping Structural Alteration of the Corpus Callosum During Brain Development and Degeneration. In: Zaidel, E.; Iacoboni, M., editors. The Parallel Brain: The Cognitive Neuroscience of the Corpus Callosum. The MIT Press; London England: 2003. p. 93-130.

Westerhausen R, Kreuder F, Dos Santos Sequeira S, Walter C, Woerner W, Wittling RA, Schweiger E, Wittling W. Effects of handedness and gender on macro- and microstructure of the corpus callosum and its subregions: a combined high-resolution and diffusion-tensor MRI study. Cognitive Brain Res. 2004; 21:418-426.

White, AM.; Swartzwelder, HS. Age-related effects of alcohol on memory and memory-related brain function in adolescents and adults. In: Galanter, M., editor. Alcohol Problems In Adolescents and Young Adults: Epidemiology, Neurobiology, Prevention, and Treatment. Springer Science +Business Media, Inc.; New York: 2006. p. 161-176.

Witelson SF. Hand and sex differences in the isthmus and genu of the human corpus callosum. Brain. 1989; 112:799-835. [PubMed: 2731030] 

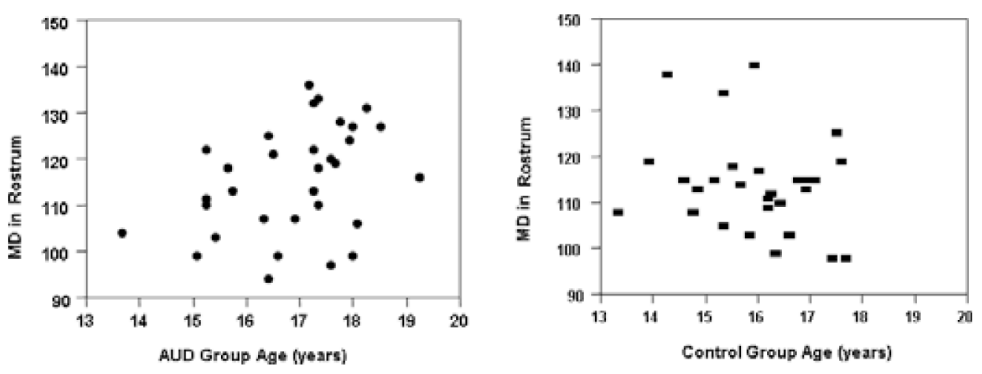

Fig. 1.

Scatterplots of the mean diffusivity (MD) values and age in the $\mathrm{CC}$ rostrum in adolescents with AUD and controls. Age was negatively associated with MD $\left(r_{\mathrm{p}}=-0.28, p<0.05\right)$ as developmentally expected in the control group but was positively associated with $\mathrm{MD}\left(r_{\mathrm{p}}=\right.$ $0.40, p<0.05)$ in the AUD group. AUD subjects are shown as solid circles and controls as solid rectangles. 

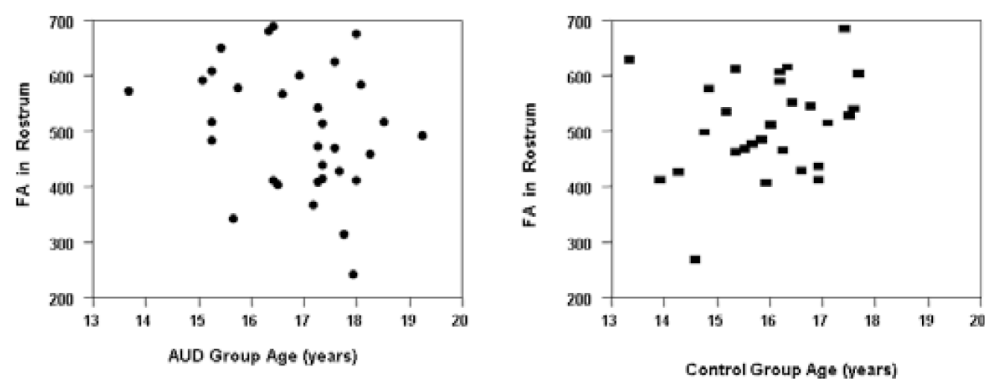

Fig. 2.

Scatterplots of the fractional anisotropy (FA) values and age in the CC rostrum in adolescents with AUD and controls. Age was positively associated with FA in the control group $\left(r_{\mathrm{p}}=0.28, p<0.05\right)$ as developmentally expected but was negatively associated with FA in the rostrum $\left(r_{\mathrm{p}}=-0.34, p<0.05\right)$ in the AUD group. AUD subjects are shown as solid circles and controls as solid rectangles. 

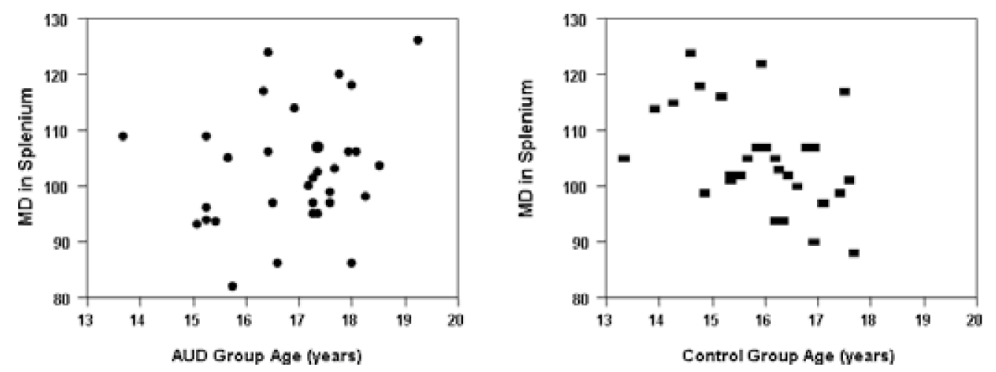

Fig. 3.

Scatterplots of the mean diffusivity (MD) values and age in the CC splenium in adolescents with AUD and controls. Age was negatively associated with $\mathrm{MD}\left(r_{\mathrm{p}}=-0.52, p<0.05\right)$ as developmentally expected in the control group but no association was seen in with MD and age in the AUD group. AUD subjects are shown as solid circles and controls as solid rectangles. 
Table 1

Demographic Characteristics of Subjects With an Adolescent Onset AUD and Healthy Comparison Subjects

\begin{tabular}{|c|c|c|c|c|}
\hline & AUD & Controls & & \\
\hline$N$ & 32 & 28 & Stats & $p$ \\
\hline Age (years) (range in years) & $16.9 \pm 1.2(13.6-19.3)$ & $15.9 \pm 1.1(13.3-17.7)$ & $t=3.1$ & 0.003 \\
\hline Sex (female/male) & $7 / 25$ & $11 / 17$ & FET & 0.17 \\
\hline Weight (lbs) & $147.0 \pm 23.3$ & $145.6 \pm 29.5$ & $t=0.21$ & 0.83 \\
\hline Height (inches) & $68.1 \pm 3.5$ & $67.5 \pm 33.9$ & $t=0.62$ & 0.54 \\
\hline Head circumference $(\mathrm{cm})$ & $56.7 \pm 1.6$ & $57.0 \pm 2.2$ & $t=0.61$ & 0.54 \\
\hline Socioeconomic status & $48.3 \pm 10.8$ & $50.5 \pm 10.0$ & $t=0.82$ & 0.42 \\
\hline Fullscale IQ & $109.2 \pm 14.6$ & $115.9 \pm 13.8$ & $t-1.79$ & $<0.08$ \\
\hline Handedness (right/left) & $29 / 3$ & $25 / 3$ & FET & 1.0 \\
\hline Birth weight (lbs) & $7.76 \pm 1.1$ & $7.76 \pm 1.3$ & $t=0.64$ & 0.52 \\
\hline Prenatal tobacco exposure (no/yes) & $18 / 14$ & $24 / 4$ & FET & 0.02 \\
\hline Prenatal alcohol exposure (no/yes) & $21 / 11$ & $22 / 6$ & FET & 0.39 \\
\hline Race (Caucasian/Africian American/Asian/Biracial) & $27 / 2 / 1 / 2$ & $21 / 4 / 0 / 3$ & $X_{2}=2.4$ & 0.50 \\
\hline Number of biological parents with a SUD history $(0 / 1 / 2)$ & $11 / 14 / 7$ & $22 / 4 / 2$ & $X^{2}=11.8$ & 0.002 \\
\hline Children's Global Assessment Scale (range) & $57.4 \pm 8.6(44-85)$ & $90.9 \pm 4.3(78-98)$ & $t=18.6$ & $<0.0001$ \\
\hline
\end{tabular}

Socioeconomic status (SES) was assessed with the Hollingshead four factor index for SES, higher values reflect upper levels of SES. 
Table 2

DTI Corpus Callosum Characteristics of Subjects With an Adolescent Onset AUD and Healthy Comparison Subjects

\begin{tabular}{|c|c|c|c|c|c|c|c|c|}
\hline \multirow[b]{2}{*}{ Corpus callosum regions } & \multicolumn{2}{|c|}{ Descriptive statistics } & \multicolumn{3}{|c|}{ Unadjusted difference } & \multicolumn{3}{|c|}{ Adjusted (age, sex) difference } \\
\hline & AUD mean (SD) & Control mean (SD) & F value & $\operatorname{Pr}>\mathbf{F}$ & Effect size & $F$-value & $\operatorname{Pr}>F$ & Effect size \\
\hline \multicolumn{9}{|l|}{ Mean diffusivity (MD) } \\
\hline Rostrum & $115.4(11.7)$ & $113.9(10.6)$ & 0.250 & 0.619 & 0.1 & 0.025 & 0.874 & 0.0 \\
\hline Genu & $109.0(10.0)$ & $109.3(8.0)$ & 0.020 & 0.887 & -0.0 & 0.263 & 0.610 & -0.2 \\
\hline Rostral body & $100.2(9.1)$ & $102.7(10.9)$ & 0.995 & 0.323 & -0.2 & 2.288 & 0.136 & -0.4 \\
\hline Anterior midbody & $95.1(11.1)$ & $95.6(9.8)$ & 0.037 & 0.847 & -0.1 & 0.285 & 0.595 & -0.2 \\
\hline Posterior midbody & $99.4(12.3)$ & $98.8(11.0)$ & 0.038 & 0.846 & 0.1 & 0.007 & 0.932 & -0.0 \\
\hline Isthmus & $105.7(13.4)$ & $111.6(11.5)$ & 3.369 & 0.072 & -0.5 & 7.276 & 0.009 & -0.8 \\
\hline Splenium & $102.7(10.7)$ & $105.0(9.2)$ & 0.819 & 0.369 & -0.3 & 0.618 & 0.435 & -0.2 \\
\hline \multicolumn{9}{|l|}{ Fractional anisotropy (FA) } \\
\hline Rostrum & $502.1(112.1)$ & $512.2(89.2)$ & 0.145 & 0.704 & -0.1 & 0.002 & 0.965 & -0.0 \\
\hline Genu & $584.2(90.6)$ & $542.8(79.9)$ & 3.476 & 0.067 & 0.5 & 3.468 & 0.068 & 0.6 \\
\hline Rostral body & $495.2(59.8)$ & $456.3(72.9)$ & 5.134 & 0.027 & 0.5 & 2.970 & 0.090 & 0.4 \\
\hline Anterior midbody & $513.2(56.1)$ & $515.4(90.1)$ & 0.013 & 0.909 & -0.0 & 0.019 & 0.892 & 0.0 \\
\hline Posterior midbody & $538.6(65.5)$ & $535.7(89.1)$ & 0.022 & 0.884 & 0.0 & 0.311 & 0.579 & 0.1 \\
\hline Isthmus & $573.6(91.3)$ & $529.8(85.5)$ & 3.648 & 0.061 & 0.5 & 10.515 & 0.002 & 0.9 \\
\hline Splenium & $635.2(66.0)$ & $620.9(100.4)$ & 0.434 & 0.513 & 0.1 & 0.328 & 0.569 & 0.1 \\
\hline
\end{tabular}

Mean diffusivity $=\mathrm{cm}^{2} / \mathrm{s} \times 10^{3}$.

Anisotropy does not have units. 